\title{
Urgences
}

\section{Donc (première notule introductive)}

\section{André Gervais et Renald Bérubé}

Numéro 19, janvier 1988

Le tour du texte

URI : https://id.erudit.org/iderudit/025440ar

DOI : https://doi.org/10.7202/025440ar

Aller au sommaire du numéro

Éditeur(s)

Urgences

ISSN

0226-9554 (imprimé)

1927-3924 (numérique)

Découvrir la revue

Citer ce document

Gervais, A. \& Bérubé, R. (1988). Donc (première notule introductive). Urgences, (19), 8-11. https://doi.org/10.7202/025440ar d'utilisation que vous pouvez consulter en ligne.

https://apropos.erudit.org/fr/usagers/politique-dutilisation/ 


\title{
Donc \\ (première notule introductive)
}

\author{
La colique vocabulique est un effet de l'in- \\ digeste nommé texte. \\ Jean-Pierre Vidal (en note), 1981 \\ Il serait temps quiun Commissaire de la \\ République des Lettres nous imposàt une \\ terminologie coherente. \\ Gérard Genette (en note), 1982
}

On peut dire, sans trop grand risque d'erreur, que la théorie du texte a pris, depuis les années 60, en Europe et en Amérique, un virage important. Ce virage se traduit, entre autres, par une prolifération terminologique de plus en plus précise qui a nécessité, à ce jour, des recensements dans quelques dictionnaires généraux et glossaires spécialisés. Les trois suivants sont actuellement couramment cités (et utilisés):

- Oswald Ducrot et Tzvetan Todorov: Dictionnaire encyclopédique des sciences du langage [1972], coll. "Points", no 110, 1979, Paris, Seuil, 474 p. (environ 800 définitions);

- Marc Angenot: Glossaire pratique de la critique contemporaine [version entièrement refondue de Glossaire de la critique littéraire contemporaine (1972)], Montréal, Hurtubise HMH, 1979, 223 p. (environ 300 définitions);

- Bernard Dupriez: Gradus. Les procédés littéraires (Dictionnaire), coll. «10/18», no 1370, Paris, UGE, 1980 (@) 1977), 543 p. (environ 1200 définitions).

Ces ouvrages - qui répertorient, au moins, des termes de la rhétorique, de la stylistique, de la linguistique, de la sémiotique, de la théorie du récit, du matérialisme historique, de l'herméneutique freudienne — datent déjà d'il y a quelques années et ne rassemblent par ailleurs que très peu des termes ( 4 ou 5 , au plus) dont le bref répertoire nous occupe ici. II n'est pas difficile de constater également qu'ils ne contiennent pas les termes forgés depuis ou encore enfouis dans des articles et livres moins lus ou publiés à l'étranger (au Québec, par exemple)'.

Nous niavons pas lintention de répertorier tous les termes manquants. Plutôt - et exclusivement, sans trop d'omissions, espérons-le - les termes théoriques et, dans quelques cas, techniques ${ }^{2}$ marqués du signe «texte», sans oublier le texte lui-même, terme qui a reçu, dès les années 60 , une voire des acceptions spécifiqueș. Coupe modeste - plus de 70 termes - et qui n'a d'autre ambition : que de donner des points de repère exacts: 
- terme ${ }^{3}$, auteur, date de la première apparition;

— définition(s) tirée(s) des articles et livres de cet auteur ou d'un autre, avec référence bibliographique complète;

- référence bibliographique complète d'un exemple d'utilisation, dans une analyse, de ce terme (s'il y a lieu).

Selon le code suivant:

— dans une définition, l'astérisque * précédant un terme (sauf le terme texte, parce qu'il est le terme de base et à cause de sa trop grande fréquence) indique que ce terme est lui-même l'objet d'une définition:

- le signe + sépare deux ou plusieurs définitions convergentes d'un terme; les précisions ajoutées par le mème auteur dans un autre livre ou article suivent, sans séparation;

- le signe $§$ sépare deux ou plusieurs définitions divergentes d'un terme;

— avant mais surtout après une définition, le signe $>>>$ annonce une notule explicative;

- les crochets [ ] encadrent un ou des mots ajoutés par nous.

Les termes recueillis sont classés dans l'un ou l'autre des quatre grands lieux du «domaine textuel». Voir les tables synthétiques correspondantes:
A. Superficies du domaine (p. 14);
B. Section intra du domaine (p. 27);
C. Section inter du domaine (p. 39);
D. Coeur du domaine (p. 52).

Il va sans dire qu'en recueillant des termes qui renvoient d'une part à des théories spécifiques (et pas nécessairement articulées l'une à l'autre), d'autre part à des pratiques qui ont souvent été publiées bien avant que tel terme soit, à leur sujet, introduit dans le champ de la théorie littéraire actuelle, ce glossaire "subit» en quelque sorte les effets de la non-hiérarchisation de l'ensemble des termes satellites de la notion de texte ainsi que du non-partage des procédures spécifiqueś qui les constituent en termes opératoires. Tout un travail reste donc à faire en ce sens ${ }^{4}$. Comme tel, cependant, il est déjà le rassemblement inédit d'un petit pan de théorie, faisant ainsi le tour du texte à sa manière, et c'est à ce titre qu'il est offert, juste retour des termes, aux théoriciens, aux analystes. Qu'il soit utile aussi aux étudiants et même aux écrivains n’est pas pour nous déplaire, au contraire.

A.G./R.B. 
1. Il faut ajouter, avec sa dizaine de termes en "texte", le récent Dictionnaire de la thérie ct de I histoire littéraires du XIXe siècle à nos jours (Paris, Retz, 1986, 335 p.) de Louis Armentier. Environ 2000 définitions.

2. Ce glossaire, on le comprendra aisément, ne tient aucunement compte des termes "poétiques" - bien que des glissements, comme on va le voir, puissent toujours advenir — faits à partir du mème radical. Il nous a été impossible de relever, dans des textes littéraires ou mème analytiques écrits en français depuis, disons, une cinquantaine d'années, tous ces néologismes. Quoiqu'un travail sur ce mot ainsi que. par exemple. sur les mots poète/poème/poésie/poétique - ici, il faudrait remonter au moins au "pohétique" de Flaubert - pourrait être fort utile pour constituer le répertoire des connivences quiont entretenues et des assauts quiont suscités (et subis) ces noms. noms de rôle et de qualité tout désignés par un métatextuel de plus en plus ciblé. Deux exemples, seulement.

Comment ne pas constater que texticule, terme forgé par Raymond Queneau en 1949 (sur le modèle mont'monticule, à nen pas douter) pour désigner certains de ses textes (recueillis depuis dans Contes et propos. Paris: Gallimard, 1981. p. 205-220). nait pas d'une part une connotation sexuelle - à cause de la proximité de "testicule" mais aussi, via les prénom et nom de celui qui l'a proposé. de la "raie". du "mont" et de la "queue" _. d'autre part la possibilité de devenir un terme d’allure générique désignant tout texte minuscule et. ainsi, entrer dans le champ des noms de textes brefs comme fragment et aphorismé, par exemple?

Veut-on, à l'autre bout du spectre, parler d'un texte qui, entremèlant langues les plus diverses et postures les plus complexes, va "à la limite", qu'on pourrait dire, à l'instar d'Hélène Cixous dans Partie (Paris. Ed. des Femmes. 1976, p. 81 - texte écrit en 1972), ce livre qui est en quelque sorte son Finnegans Wake. qu'il est (un, le) textrême.

3. Chaque fois qu il a été possible, nous avons uniformisé en agglutinant le préfixe. II reste donc peu de termes avec un trait d'union. Côté néologie, nous constaterons rapidement les points suivants.

La quasi-totalité des termes de ce petit glossaire est issue du processus de la dérivation, c'est-à-dire de l'sagglutination d'élèments lexicaux, dont un au moins n'est pas susceptible d'emploi independant, en une forme unique" (dit le Dictionnaire de linguistique de Jean Dubois et al.. Paris, Larousse, 1973. p. 141). II y a donc:

- une trentaine de préfixes dorigine grecque (anti-, archi-, auto-, epi-, hétéro-, homo-, etc.), latine (colnl-, contre-, extra-, infra-, inter-, intra-, etc.) et française (après-, avant-, hors-, contre-) ainsi qu une dizaine d'élements d'origine grecque (biblio-, bio-, calli-, gramma-, etc.) ou française (anthologico-, mimo-) mis en position de préfixe, joints principalement au nom "texte»;

- quelques suffixes ajoutés au nom "contexte" (-ure), aux adjectifs "textuel" et "textillel" (-ité) et au verbe "textualislerl" (-ation) ainsi que les néologismes suivants qui trouvent, étant données la date de leur apparition et leur définition, leur "modèle" dans tels mots déjà existants: textolecte (-lecte d'après dialecte), textologie (-logie d'après biologie), textanalyse (-analyse d'après psychanalyse), textuation (-uation d'après situation).

Un seul terme répondrait exactement au processus de la composition, c'est-à-dire à "la formation d'une unité sémantique à partir d'éléments lexicaux susceptibles d'avoir par eux-mèmes une autonomie dans la langue» (Dictionnaire de linguistique. p. 109): ce terme est milletexte. Mais il faut souligner les limites dune telle opposition entre dérivation et composition: dans textanalyse, par exemple, l'autonomie des composants n'est pas moindre que dans milletexte.

Par ailleurs, il va sans dire qu un certain nombre de ces termes ont été forgés ponctuellement pour les besoins d'une analyse, alors que plusieurs autres — ceux 
de Jean Ricardou et de Gérard Genette, particulièrement - sont partie prenante d'un "système" qui. s'élaborant depuis plusieurs années, tend nettement à construire sa propre terminologie, soit par création de nouveaux termes (néologismes lexicaux), soit par déplacement de significations (néologismes sémantiques). Ceci permet d'expliquer dune part le fait que certains termes ne soient actualisés que sous une forme dite concrète (callitexte, par exemple). d'autres sous une,forme dite abstraite (grammatextualité, par exemple). d'autres enfin sous ces deux formes (comme très souvent chez Genette: architexte et architextualité. par exemple), d'autre part la non-«harmonisation" de l'ensemble. Voir. sur ce dernier point. la note 4 .

4. Ce travail, à peine amorcé devrait rendre compte, entre autres, des différences réelles sous les ressemblances. des imprécisions, des recoupements, des inféodations et, bien sûr, des trous. Deux exemples, très rapidement pointés.

Contretexte et antitexte ont des préfixes synonymiques. Or le premier terme est nettement une particularisation du couple hypotexte/hypertexte (qui fait partie de la section $C$, dite section inter du domaine) alors que le second est plutôt en opposition partielle au texte, terme générique (de la section $D$, dite coeur du domaine).

Prototexte et pré-texte désignent des configurations (de l'ordre de l'inconscient d'une part, des conditions de production d'autre part) qui sont canalisées, focalisées de plus en plus par et vers le texte, et ce en opposition aux configurations de l'extratexte et du hors-texte. Mais ne faut-il pas ajouter que ces quatre termes, bien que marqués du signe " $\quad$-texte», s'opposent absolument au texte en ce que, littéralement, ils ne le désignent pas (prototexte, par exemple, étant bien défini comme un agrégat d'images antérieures à ('écriture), au contraire de l'avant-texte qui, littéralement, est dans le texte.

Tout cela, bien sûr, demande à être précisé, développé, argumenté, voire même rectifié. 